\title{
Eco Evidence: using the scientific literature to inform evidence-based decision making in environmental management
}

\author{
$\underline{\text { J.A. Webb }}^{\text {a,b }}$, S.R. Wealands ${ }^{\text {a,c }}$, P. Lea ${ }^{\text {a,c }}$, S.J. Nichols ${ }^{\text {a,d }}$, S.C. de Little ${ }^{c}$, M.J. Stewardson ${ }^{\text {a,c }}$ and \\ R.H. Norris ${ }^{\text {a,d }}$ \\ ${ }^{a}$ eWater Cooperative Research Centre \\ ${ }^{b}$ Department of Resource Management \& Geography, The University of Melbourne, Victoria \\ ${ }^{c}$ Department of Infrastructure Engineering, The University of Melbourne, Victoria \\ ${ }^{d}$ Institute for Applied Ecology, University of Canberra, Australian Capital Territory \\ Email: angus.webb@unimelb.edu.au
}

\begin{abstract}
In the face of increasing human-induced pressures on natural environments, managers must balance the needs of environmental and human uses in a transparent and defensible manner. Sound decision making in environmental management relies on understanding causal relationships between environmental stressors and ecological responses. However, causal relations are difficult to demonstrate in natural environments because of the difficulty of performing experiments, natural variability, lack of replication, and the presence of confounding influences. Partly because of this, most environmental management decisions are made using expert opinion. Such decisions can lack transparency. Epidemiologists recognized similar difficulties in ascribing causality in the 1960s, and developed 'causal criteria' to assess causal relations in epidemiological investigations. Causal criteria analysis builds a case for causality based on the cumulative strength of many individually weak pieces of evidence.
\end{abstract}

There have been several calls to use causal criteria analysis in environmental science, but few case studies exist. This may partly result from the lack of standardized methods and analysis tools (analogous to statistical software). We describe the Eco Evidence software package, which has been developed to facilitate causal criteria analysis in environmental science. It employs the published scientific literature as a previously underused source of evidence for such analyses. The software consists of a web database application - the Eco Evidence Database - for storing and sharing 'evidence items' (the information extracted from individual studies necessary for the causal criteria analysis); and a desktop analysis tool - the Eco Evidence Analyser that uses evidence shared via the web application to assess causal hypotheses. The database provides a permanent, online repository for causal evidence, accessible with any web browser. Moreover, it allows users to access evidence items entered by previous users, thereby reducing the burden of extracting evidence from the literature. The analysis tool uses a wizard-style interface to guide users through an 8-step standardized approach to causal criteria analysis specifically designed for use in the environmental sciences. A full report is produced at the end of the assessment, which contains all the information used to reach the conclusion. This maximizes transparency of the assessment, and means that any bias in the review will be detected more easily compared to a traditional literature review. We demonstrate the Eco Evidence approach with an example that investigates the evidence for the question of whether increased base flows and increased frequency of high flow events can reduce the encroachment of terrestrial vegetation into the channels of regulated rivers.

Legislative and social imperatives are prompting a move from an experience-based to an evidence-based model of environmental management. This will lead to more transparent and repeatable decisions, and potentially better decisions overall. However, such a major change of practice will not be easily achieved. Tools such as Eco Evidence will facilitate the transformation by assisting managers to use scientific evidence to inform difficult decisions. With the Eco Evidence software now publicly released and freely available, we are turning our attention to facilitating uptake of the method through promotion and training. We expect that early adopters of Eco Evidence will help to drive rapid evolution of the method and software. However, the current version is already sufficiently well-developed to aid environmental science and management.

Keywords: Systematic review, Database, Analysis software, Causal Criteria Analysis, Decision making 


\section{INTRODUCTION}

\subsection{Weak inference in environmental sciences}

Environmental investigations are carried out in conditions where it is often difficult or impossible to develop strong inferences concerning hypothesized causes and effects (Beyers, 1998). This mainly occurs because the experimental design of such investigations usually lacks of one or more the characteristics that can serve to increase our confidence that a result indicates a cause-effect relationship (Downes et al., 2002). At the studydesign level, the 'treatments' are often not randomly allocated. It is also common to: i) have no data to describe a location before the hypothetical disturbance, ii) be unable to allocate control locations, iii) be faced with confounding environmental factors, and iv), suffer from a lack of adequate replication. Thus, it may be argued that very few studies of human impacts on the environment can provide a 'severe' test (sensu Popper, 1983) of a hypothesized cause-effect relation. However, developing strong cases for causal relationships in natural environments is of great importance, especially for management. Controversial management decisions, often involving large expenditure of taxpayer funds, must be transparent and defensible in their use of 'best available science' (Ryder et al., 2010).

If environmental studies can seldom establish causality, then additional evidence is needed (Downes et al., 2002). Such evidence can include repeated studies of the same hypothesized cause-effect relation in different environments and with different study designs and methods; supporting experimental results from smallscale manipulations in the laboratory or field; or evidence of the hypothesized causal agent within the target organism (e.g. body burden of heavy metals in fish near mine sites). Individually, none of these types of evidence may be convincing, but when considered together, they may amount to a powerful argument for causality. Intentionally or otherwise, investigators often seek to strengthen their arguments for causality through the informal inclusion of multiple lines of evidence in the discussion sections of their research papers (reviewed in Downes et al., 2002). However, what has been lacking is a rigorous framework for synthesising this evidence to assess causality in environmental research.

\subsection{Causal criteria for causal inference}

Faced with similar problems in identifying cause-effect relationships, epidemiologists developed 'causal criteria' analysis in the 1960s (Tugwell and Haynes, 2006). The causal criteria are a checklist; the evidence for each hypothesized cause-effect relation is assessed against several criteria to assess how well it supports an argument for causality. The best-known set of epidemiological causal criteria was developed by Hill (1965), but the criteria have been widely debated and revised since then (e.g. Susser, 1991). Suter et al. (2010) provide a good introductory review to research into causality. Several calls to use causal criteria in environmental sciences (e.g. Beyers, 1998; Downes et al., 2002) have produced few case studies, and, those case studies have used inconsistent definitions of the criteria and methods for combining evidence. Standardized methods and tools may aid uptake of causal criteria in environmental sciences.

\subsection{The Eco Evidence analysis framework}

We have developed an 8-step framework, named Eco Evidence, based on the epidemiological approach (Figure 1). The method operates within the conjecture-refutation model of scientific progress familiar to most researchers, and can also identify knowledge gaps. It centers on systematic review of the literature, as an underused source of knowledge. Each study is weighted by its ability to contribute to the argument for causality, and studies are considered collectively

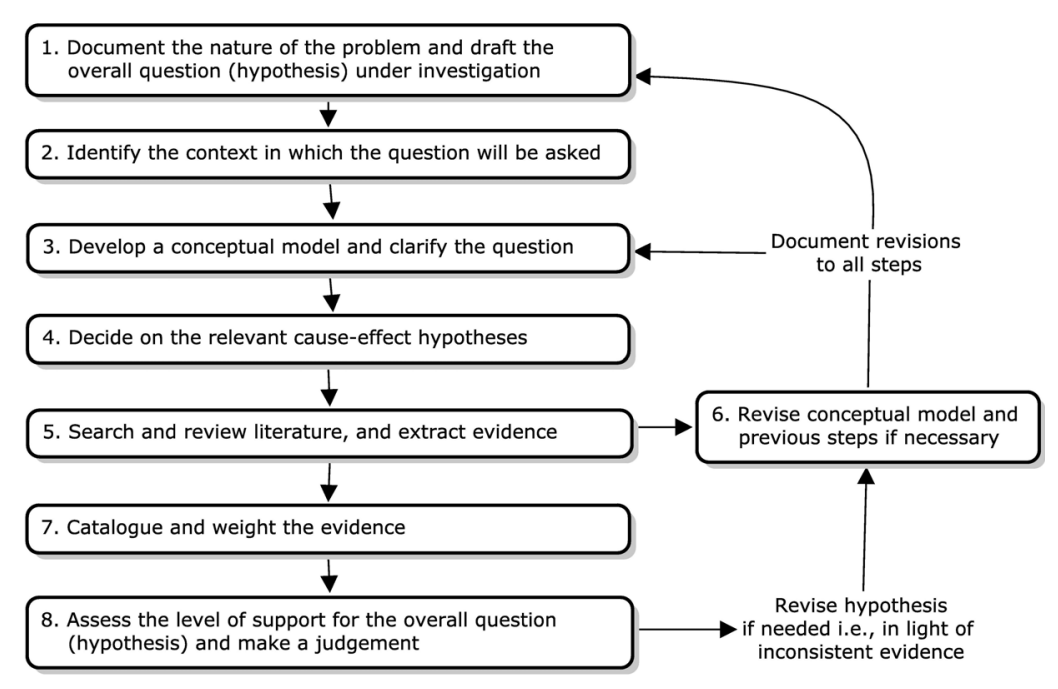

Figure 1. The Eco Evidence framework (reprinted from Norris et al., 2012). 
against one or more causal hypotheses. Steps 1-4 and 6 center on problem formulation. The reviewer documents the nature of the problem under investigation and formulates an initial question, identifies the context in which the question will be asked, develops a conceptual model of the problem, documents the hypothesized cause-effect relationships, and if necessary revises steps $1-4$ following review of the literature (Step 6). Step 5 consists of the literature search and systematic review, and is the single largest task within the framework. Next, the evidence is extracted from the relevant articles and collated (see detail below). In steps 7 and 8 , the evidence is weighted, combined, and considered to assess the level of support for and against the individual causal hypotheses, which are then assessed collectively to inform an overall finding for the original question. The framework is presented in full detail in Norris et al. (2012) and Nichols et al. (2011). Discussions of its strengths, weaknesses, and ideas for improvement are included in Norris et al. (2012) and Webb et al. (in press). The purpose of this paper is to describe the supporting Eco Evidence software package.

\section{ECO EVIDENCE SOFTWARE}

Eco Evidence can be obtained online at www.toolkit.net.au/tools/eco-evidence. The software consists of two components: a database for storing and sharing 'evidence items' (information on hypothesized cause and effect, and experimental design) from publications, and a desktop analysis tool to guide users through the 8step process to assess the level of support for causal hypotheses.

\section{1. $\quad$ Eco Evidence Database (EED)}

The Eco Evidence Database (hereafter EED) is an online database application for storing and sharing evidence items. It provides a permanent repository for causal evidence items and allows users to access and re-use evidence items entered by other users. It is accessible via any web browser and requires no installation or system customization, making it easy for new users to contribute, find and retrieve evidence.

\section{Data structure}

The key entities managed in the database are citations and evidence items. The information is organized such that a citation can have one or more items of evidence. Along with each of these entities, there are a number of standard fields collected that identify and describe it. For example, a citation stores the common fields of title, author, year, etc., but users are also prompted to classify the citation's spatial and temporal scale, region, and ecosystem/climate type. These extra classifications can be used to filter the evidence returned from a database search to, for example, a specific spatial context. The standard attributes captured for each evidence item belong to several categories (Table 1), and have been determined over the past two years through user input, usage of the data, and discussions with collaborators. To date, these attributes have been extracted for over 1000 evidence items (from over 400 papers) and have proven sufficient for causal criteria assessment in a number of case studies (see Webb et al., in press).

Table 1. Main categories of attributes collected for an evidence item.

\begin{tabular}{ll} 
Cause / effect & $\begin{array}{l}\text { A classification and detailed description of the putative cause and effect is required, along with a trajectory } \\
\text { describing the nature of the change observed }\end{array}$ \\
$\begin{array}{l}\text { Study design } \\
\text { Association }\end{array}$ & $\begin{array}{l}\text { Classifications of the type and form of the association described between the cause and effect } \\
\text { Analysis }\end{array}$ \\
Strength & Descriptions of how appropriate the analysis and interpretations conducted were considered to be \\
Other & Classifications of how strong the association was and how that compared with background variability \\
\hline
\end{tabular}

For many of the evidence attributes, classifications (i.e. drop-down lists) are used because the various options have specific interpretations and weightings applied within the Analyser tool. For the cause and effect, users provide both a classification (from a standard term list) and also a free-text description of the cause. The standard term list covers the scope of applications Eco Evidence is suited to, but is open to further revision and expansion. For each term, a definition is available to help users classify cause and effect for a particular item of evidence. This term list plays a key role in retrieving evidence on any cause-effect linkage, which in turn forms the building blocks of the analysis conducted with the Analyser.

\section{Functionality}

The database allows users to view and edit existing evidence and citations, as well as exporting a collection of evidence items to a .csv (comma separated values) text file. Evidence from the database can also be 
retrieved directly into the Analyser via a web service (see below). When exporting evidence, a 'shopping cart' is available for users to build up a list of relevant evidence and citations that can then be exported. All registered users can search and retrieve content from the database, as well as adding new citations and evidence. All modifications are tagged with the user name, as a means of basic quality control over the changes made. Basic users are permitted to edit and refine their own contributions, while 'power' users have the ability to update all evidence items to make amendments.

\subsection{Eco Evidence Analyser (EEA)}

The Eco Evidence Analyser (hereafter EEA) is a Windows-based software tool that uses a wizard-style interface to guide users through the 8 step framework described above. It requires installation on the client machine, but connects to remote web services (using the Windows Communication Framework - WCF) for retrieving evidence items from the EED. The citation and evidence data are stored within a structured XML project file, with separate project files recommended for each causal analysis conducted.

The analysis focuses on evaluating the evidence for one or more cause-effect linkages, with the evidence items for each linkage providing the grounds for assessment. Each linkage can be thought of as a link in a conceptual model, with trajectories describing what the user expects to happen in the system (e.g. an increase in surface water leads to an increase in vegetation growth). The attributes stored in and retrieved from the database provide most of the information required for the analysis. However, the user is required to confirm whether each evidence item retrieved by the search is relevant to their hypothesis. Relevance may be based on, for example, spatial or climatic context of the evidence item, the type of ecosystem studied, or whether research is restricted to undisturbed sites. Once this is completed, a weight is determined for each evidence item based on study type and sample replication (Norris et al., 2012).

After the evidence has been collated and weighted, the software automatically compares the trajectories (i.e. increase, decrease, or change) of the hypothesized linkage to the collated evidence. For example, if the evidence item reported decreased vegetation growth in response to an increase in surface water, the response would be considered as evidence against the hypothesized linkage described earlier. Based on this comparison, each item of evidence contributes to either supporting or rejecting the hypothesis. The weighted values for each study are summed and compared against a threshold to give an overall evaluation of the strength of evidence for and against each linkage. The Eco Evidence Analyser undertakes the weighting and summation automatically, allowing the user to focus on interpreting the evidence. Users may modify the weightings and thresholds if such a change is justified. The software produces a final report that presents the information input by the user (question, context, conceptual model, search strategies), the findings for each causal hypothesis/linkage, the evidence used to reach those conclusions, and the sources of that evidence. This report provides complete transparency for the causal assessment, and means that any bias (either deliberate or inadvertent) will be more easily detected than with a traditional literature review.

\section{ILLUSTRATIVE CASE STUDY}

We provide a case study to illustrate the application of the framework and software. However, it is greatly abbreviated and contains far less detail than a complete systematic review using Eco Evidence. For fullydeveloped case studies, readers are directed to Harrison (2010) and Greet et al. (2011).

Many regulated rivers in south-eastern Australia suffer from encroachment of terrestrial and exotic vegetation into the channel. Environmental flow assessments commonly predict that restoring a more natural level of base flow in winter/spring and/or re-instigating higher flow events will reduce encroachment (e.g. Chee et al., 2009). We used Eco Evidence to examine the evidence for this proposition.

Step 1 - The question: Will increased winter/spring base flows and more frequent high flow events lead to reductions in terrestrial plant encroachment into river channels?

Step 2 - The context: We were interested in generalising conclusions to lowland regulated rivers in southeastern Australia. This does not mean, however, that only evidence from such systems may be used.

Step 3 - Conceptual model: Increased base flows and increased frequency of high flow events will lead to increased inundation of channel features currently colonized by terrestrial and exotic vegetation. Reduced vegetation encroachment may be achieved via any or all of the mechanisms of: reduced germination of terrestrial propagules within the channel, dieback of existing vegetation within the channel, and physical removal of such vegetation from the channel through flows that scour the bed (Figure 2). For this demonstration, we assessed the biological links \#1 and \#2 indicated by blue arrows. Green arrows were not assessed. Black arrows can be assumed. 
Step 4 - Identify causes and effects: We chose terms from the standard terms list in Eco Evidence (Table 2). Because changes in flow regimes can be quantified in several ways, we also used an increase in surface water (depth) and surface water (volume) as causes. Two effect terms, vegetation (reproduction) and vegetation (germination) were used to test link \#1 because both terms exist in the EED.

Step 5 - Conduct literature search and review: We searched the EED using the EEA web service on 25 June, 2011 using all combinations of cause and effect terms. The search retrieved 107 previously-existing evidence items. Upon examination, 58 of these were deemed relevant to the question of flow effects upon 'terrestrial' species.

Step 6 - Revise conceptual model if necessary: Many of the evidence items were from empirical studies that linked inundation of channel features directly to reduced abundance of terrestrial vegetation (red link \#3 in Figure 2), and so this was added as an additional link to be tested. Similar to link \#1, this link was tested by multiple

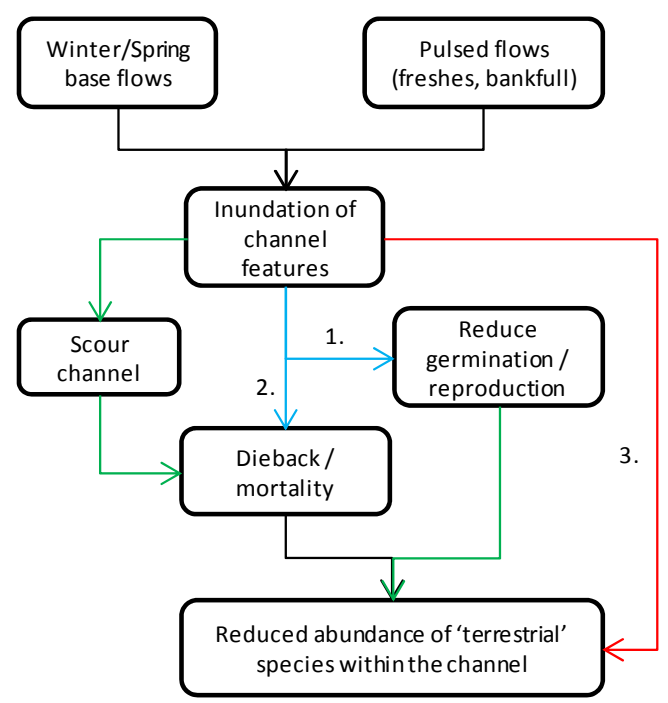

Figure 2. Conceptual model of flow effects on in-channel 'terrestrial' vegetation. hypotheses because of multiple relevant terms in the EED.

Step 7 - Catalogue and weight the evidence: The EEA software makes this step implicit. As soon as an evidence item is deemed relevant to a hypothesis, its evidence weight is recorded either in support of that hypothesis or against it ('Response' and 'Consistency' criteria Table 2; see Norris et al., 2012 for full explanation). If a user wished to change the weightings or threshold used in the analysis, it would be done at this stage, but here we used the default weights (given in Norris et al., 2012). At this step we combined evidence recorded against the three attributes of surface water (depth, volume, frequency), because we were satisfied that the relevant evidence for all of these pertained to inundation of channel features.

Step 8 - The judgment: There was insufficient evidence to test hypothesis \#5, indicating incomplete coverage of the EED or a research gap (Table 2). The evidence for hypothesis \#2 was inconsistent (both for and against), indicating that the hypothesis was not specific enough (Table 2). The reviewer should then examine the evidence to identify the underlying reasons (e.g. Greet et al., 2011), and revise the hypothesis (Norris et al., 2012). Other hypotheses were supported (Table 2). The final judgment in an Eco Evidence analysis regarding the overall question developed at Step 1 requires an intelligent synthesis of the conclusions for each individual hypothesis (Norris et al., 2012). While not all of our individual hypotheses were supported, each link was. Our assessment is that there was sufficient evidence to conclude that increased flows should reduce encroachment of terrestrial vegetation, with a reduction in germination and an increase in inundation-induced mortality being possible direct causal mechanisms.

Table 2. Conclusions of the Eco Evidence analysis for each of the case-study hypotheses. 'Response' and 'Consistency' columns are the summed evidence weights in favour of, and against, the causal hypothesis.

\begin{tabular}{clcccc}
\hline$\#$ & Causal Hypothesis & Link \# & Response & Consistency & Conclusion \\
\hline 1 & $\uparrow$ surface water (frequency) $\rightarrow \downarrow$ vegetation (germination) & 1 & 59 & 16 & Support for hypothesis \\
2 & $\uparrow$ surface water (frequency) $\rightarrow \downarrow$ vegetation (reproduction) & 1 & 29 & 22 & Inconsistent evidence \\
3 & $\uparrow$ surface water (frequency) $\rightarrow \uparrow$ vegetation (mortality) & 2 & 20 & 7 & Support for hypothesis \\
4 & $\uparrow$ surface water (frequency) $\rightarrow \Delta$ vegetation (assemblage) & 3 & 123 & 17 & Support for hypothesis \\
5 & $\uparrow$ surface water (frequency) $\rightarrow \downarrow$ vegetation (exotic invasion) & 3 & 5 & 0 & Insufficient evidence \\
6 & $\uparrow$ surface water (frequency) $\rightarrow \downarrow$ vegetation & 3 & 76 & 17 & Support for hypothesis \\
\hline
\end{tabular}

\section{DISCUSSION}

Currently, many environmental management decisions are informed by the experience of the manager or by expert advice (Stewardson and Webb, 2010). Such decisions may lack transparency. Systematic synthesis of evidence used to inform decisions will greatly improve their transparency and defensibility. Moreover, moving towards a more 'evidence-based' approach to environmental management may lead to improved environmental outcomes (see Pullin et al., 2009). Developing standards and tools for evidence-based practice in environmental science should help to facilitate such a transition. Eco Evidence was developed in response to the observation that there is a huge amount of knowledge within the scientific literature that is currently 
under-utilized. In fact, this literature 'deluge' may now be constraining scientific progress rather than aiding it (Attwood et al., 2009), and tools like Eco Evidence are required to more effectively summarise the research in systematic reviews. Currently, systematic review is an underused technique in environmental science (Pullin et al., 2009). Traditional 'narrative' literature reviews tend to summarize a large body of literature, but seldom attempt to address questions within any analytic framework. In the context of Eco Evidence, narrative reviews seldom ask the question of whether there is 'enough' evidence to reach a conclusion concerning a causal relation between an environmental stressor and observed effect.

The version 1 release of Eco Evidence is expected to spur further development of the method and software. While we have successfully used the approach in a number of case studies (Webb et al., in press), these have generated a number of ideas about how both method and software might be improved. With the public release of the software, and anticipated uptake by researchers and managers beyond our research group, we expect this wish-list to grow rapidly. For the Eco Evidence software, changes within the first 12 months of release could include: i) write-back capability to EED from EEA where the analysis software would become a secondary interface for adding evidence to the online database, rather than simply a tool for retrieving and analyzing evidence; ii) establishing an online repository of Eco Evidence analysis reports similar to the way that the Collaboration for Environmental Evidence (www.environmentalevidence.org) maintains a library of systematic reviews; and iii) storing working EEA projects online, and allowing multiple users to collaborate on them, similar to USEPA's Interactive Causal Diagram tool (www.epa.gov/caddis/cd_icds intro.html).

A key feature of Eco Evidence is that it provides access to a reusable bank of evidence that can be used to analyze a new question, or to repeat a previous review when new evidence becomes available. However, for Eco Evidence to realize its full potential, the database needs to be populated with far more evidence than it currently contains, and kept up to date with evidence from newly published literature. To date, evidence has been manually extracted by researchers undertaking systematic reviews on specific questions. However, this is a labour-intensive process that leads naturally to fairly specialized areas of coverage (i.e. the database currently has over 400 evidence items linking changes in river flow regimes to vegetation responses) rather than a broad coverage of topics that would be of maximum use for environmental science and management.

We have identified three potential pathways for larger-scale population of the database. First, studies in the database will have a better chance of being cited in review articles than other studies. This may provide incentive for researchers to input evidence from their own publications. Second, there is potential to enter into arrangements with publishers such that evidence is uploaded for publications appearing in certain journals. The potential for increased citation rates and impact factors may provide incentive for publishers to become involved. Third, the artificial intelligence technique, Natural Language Processing has successfully been used to extract knowledge from the medical literature to assist clinical decisions (Demner-Fushman et al., 2009), and may be useful for at least partly automating the extraction of evidence from the extensive back catalogue of literature. An alternate path for larger-scale uptake of Eco Evidence would be if it were compatible with an international standard for ecological evidence storage and/or synthesis. We are working with the USEPA, who regularly extract and analyze evidence from the scientific literature using their CADDIS framework (www.epa.gov/caddis). This collaboration has established a draft standard definition of an evidence item and web services are running that allow retrieval of evidence from both the USEPA's CADDIS database and the EED. Moving forward, these standards will enable greater access to evidence for use in analyses and help to avoid duplication during evidence extraction.

Whatever the means of database population, quality control of the evidence is an issue yet to be fully addressed. In a peer-produced database, evidence items could receive user ratings, similar to those on many social networking and e-commerce sites. Similarly, management agencies using evidence from the database may 'stamp' evidence items they deem of sufficient quality to use in their decision making processes. For the moment, however, the source of all evidence is fully acknowledged, and users should check the quality of evidence downloaded from the database against the original sources.

Products and methods for evidence-based practice, such as Eco Evidence, are seeking to drive a change in practice in environmental science and management. This challenge cannot be understated. Early adopters of the Eco Evidence method and software have included students, staff in our research groups, and industry personnel with whom we are collaborating. As Eco Evidence moves from development to an adoption phase, training will be available at conference workshops, through management agencies, and online.

\section{CONCLUSIONS}

Eco Evidence provides a means for both researchers and managers to synthesize the vast scientific literature to assess questions of cause and effect in natural environments, thereby using the 'best available science' to 
inform 'evidence-based' decision making. There are legislative imperatives to facilitate uptake of the method by management agencies, and it is also useful for systematic reviews on both site-specific and more general questions in environmental science. While the version 1 software and method are likely to evolve considerably over the next few years in response to user feedback, they are well-developed and ready for immediate use. The focus of our work will now turn to facilitating uptake of the method.

\section{ACKNOWLEDGMENTS}

The development of Eco Evidence was funded by the eWater Cooperative Research Centre. The case study presented here was funded by Australian Research Council Linkage Project LP100200170.

\section{REFERENCES}

Attwood, T.K., D.B. Kell, P. McDermott, J. Marsh, S.R. Pettifer, and D. Thorne (2009). Calling International Rescue: knowledge lost in literature and data landslide! Biochemical Journal, 424, 317-333.

Beyers, D.W. (1998). Causal inference in environmental impact studies. Journal of the North American Benthological Society, 17(3), 367-373.

Chee, Y.E., J.A. Webb, M. Stewardson, and P. Cottingham (2009). Victorian Environmental Flows Monitoring and Assessment Program: Monitoring and assessing environmental flow releases in the Thompson River. eWater Cooperative Research Centre, Canberra. Available from http://tinyurl.com/VEFMAP-Thomson.

Demner-Fushman, D., W.W. Chapman, and C.J. McDonald (2009). What can natural language processing do for clinical decision support? Journal of Biomedical Informatics, 42(5), 760-772.

Downes, B.J., L.A. Barmuta, P.G. Fairweather, D.P. Faith, M.J. Keough, P.S. Lake, B.D. Mapstone, and G.P. Quinn (2002). Monitoring Ecological Impacts: Concepts and Practice in Flowing Waters, pp. xii, 434, Cambridge University Press, Cambridge.

Greet, J., J.A. Webb, and R.D. Cousens (2011). The importance of seasonal flow timing for riparian vegetation dynamics: a systematic review using causal criteria analysis. Freshwater Biology, 56(7), 12311247.

Harrison, E.T. (2010). Fine sediment in rivers: scale of ecological outcomes. Ph.D. Thesis, University of Canberra, Canberra. Available from http://tinyurl.com/Harrison-2010.

Hill, A.B. (1965). The environment and disease - association or causation? Proceedings of the Royal Society of Medicine-London, 58(5), 295-300.

Nichols, S., A. Webb, R. Norris, and M. Stewardson (2011). Eco Evidence analysis methods manual: a systematic approach to evaluate causality in environmental science. eWater Cooperative Research Centre, Canberra. Available from http://tinyurl.com/Eco-Evidence-manual.

Norris, R.H., J.A. Webb, S.J. Nichols, M.J. Stewardson, and E.T. Harrison (2012). Analyzing cause and effect in environmental assessments: using weighted evidence from the literature. Freshwater Science, 31(1), doi: 10.1899/1811-1027.

Popper, K.R. (1983). Realism and the aim of science: Postscript to the logic of scientific discovery, pp. xxxix, 420, Hutchinson, London.

Pullin, A.S., T.M. Knight, and A.R. Watkinson (2009). Linking reductionist science and holistic policy using systematic reviews: unpacking environmental policy questions to construct an evidence-based framework. Journal of Applied Ecology, 46(5), 970-975.

Ryder, D.S., M. Tomlinson, B. Gawne, and G.E. Likens (2010). Defining and using 'best available science': a policy conundrum for the management of aquatic ecosystems. Marine and Freshwater Research, 61, 821828.

Stewardson, M.J., and J.A. Webb (2010). Modelling ecological responses to flow alteration: making the most of existing data and knowledge, In Saintilan, N. and I. Overton (Eds.), Ecosystem Response Modelling in the Murray-Darling Basin, pp. 37-49. CSIRO Publishing, Melbourne.

Susser, M. (1991). What is a cause and how do we know one - a grammar for pragmatic epidemiology. American Journal of Epidemiology, 133(7), 635-648.

Suter, G.W., S.B. Norton, and S.M. Cormier (2010). The science and philosophy of a method for assessing environmental causes. Human and Ecological Risk Assessment, 16(1), 19-34.

Tugwell, B., and R.B. Haynes (2006). Assessing claims of causation, In Haynes, R.B., D.L. Sackett, G.H. Guyatt and P. Tugwell (Eds.), Clinical Epidemiology: How to do Clinical Practice Research, pp. 356-387. Lippincott Williams \& Wilkins, Philadelphia.

Webb, J.A., S.J. Nichols, R.H. Norris, M.J. Stewardson, S.R. Wealands, and P. Lea (in press). Ecological responses to flow alteration: assessing causal relationships with Eco Evidence. Wetlands. 